\title{
SASOCP position statement on the pharmacist's role in antibiotic stewardship 2018
}

\author{
Schellack N,' Bronkhorst E,, ${ }^{2}$ Coetzee R, ${ }^{3}$ Godman B, ${ }^{4}$ Gous AGS, ${ }^{5}$ Kolman S, ${ }^{6}$ Labuschagne $Q,{ }^{2}$ Malan $L,{ }^{2}$ Messina AP, \\ Naested C, ${ }^{9}$ Schellack $G,{ }^{10}$ Skosana $P^{2}$ Van Jaarsveld ${ }^{11}$
}

\author{
${ }^{3}$ Senior Lecturer, School of Pharmacy, Faculty of Natural Sciences, University of the Western Cape \\ Pharmacology, Karolinska Institutet, Stockholm, Sweden \\ ${ }^{6}$ Clinical Pharmacist Nelson Mandela Children Hospital \\ ${ }^{7}$ Department of Pharmacy, Netcare Hospitals Ltd, Johannesburg, South Africa \\ ${ }^{8}$ School of Therapeutic Sciences, Faculty of Health Sciences, University \\ of the Witwatersrand. \\ ${ }^{9}$ Pharmacist, Netcare Greenacres Hospital \\ ${ }^{10}$ Pharmaceutical Industry, South Africa \\ ${ }^{11}$ Clinical Pharmacy Specialist Mediclinic Southern Africa.
}

${ }^{1}$ Associate Professor, School of Pharmacy, Faculty of Health Sciences, Sefako Makgatho Health Sciences University

${ }^{2}$ Lecturer, School of Pharmacy, Faculty of Health Sciences, Sefako Makgatho Health Sciences University

${ }^{4}$ Professor, Strathclyde Institute of Pharmacy and Biomedical Sciences, Strathclyde University, Glasgow, UK and Division of Clinical

${ }_{5}^{5}$ Professor/Acting Dean, School of Pharmacy, Faculty of Health Sciences, Sefako Makgatho Health Sciences University

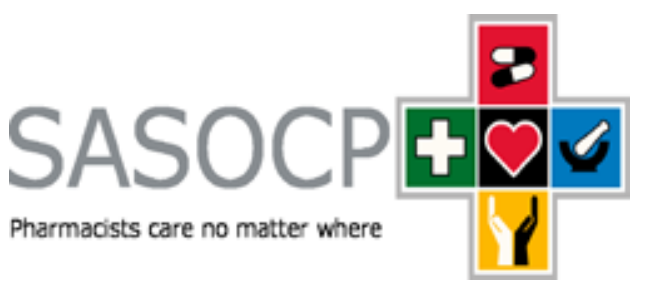

Antibiotics are the most commonly prescribed medicines in global healthcare practice today. Their effectiveness is crucial and often life-saving in humanity's battle against pathogens and infectious diseases. Antibiotic/antimicrobial stewardship strategies and programmes have become vital to the preservation of effective antibiotics and the optimisation of their use. The South African Society of Clinical Pharmacy (SASOCP) has written this guideline to outline the importance, role and purpose of pharmacists in such stewardship programmes, both in the public, as well as the private hospital sectors in South Africa. It also provides an overview of various approaches to antibiotic preservation, behavioural change, stewardship measures, and monitoring strategies.

\section{Aim}

The South African Society of Clinical Pharmacy (SASOCP) has written this guideline to outline the importance, role and purpose of pharmacists in antibiotic stewardship programmes, in both public and private hospitals in South Africa.

Antibiotics are the most commonly prescribed medicines in both the community and hospital health care setting and are crucial, life-saving medicines in the fight against infectious diseases. ${ }^{1-4}$ Antibiotic utilisation world-wide has increased by $36 \%$ between 2000 and 2010, with countries such as China, India, and South Africa accounting for $76 \%$ of this increase. ${ }^{4,5}$ However, the increasing use of antibiotics, including their misuse, has escalated the prevalence of antimicrobial resistance (AMR).

This poses a devastatingly serious threat to public health as well as increasing costs, ${ }^{6-9}$ with the rise in AMR potentially resulting in AMR becoming a leading cause of death worldwide by 2050, reducing GDP by $2 \%$ to $3.5 \%$ and costing up to US\$ 100 trillion per year. ${ }^{6,10}$ This requires urgent action across all countries and government sectors to reduce the imminent threat. ${ }^{6}$ Importantly, factors contributing to the rise of AMR include: incorrect selection of an appropriate agent, incorrect timing of administration, insufficient dosing and incorrect duration of therapy. ${ }^{11}$

The role of the antibiotic stewardship pharmacist includes the preservation of antibiotics through the monitoring, evaluation and guidance of appropriate antibiotic use. This includes review of the so-called 4 Ds of optimal antibiotic therapy: right Drug, right Dose, De-escalation to pathogen-directed therapy and the right Duration of treatment. This aligns to the aim of antibiotic stewardship (ABS), which, by definition, is "an ongoing effort to optimise antibiotic use in order to improve patient outcomes, ensure cost-effective therapy and reduce the adverse consequences of antibiotic usage, which includes antimicrobial resistance".12

\subsection{Antibiotic stewardship in South Africa (SA)}

Currently, novel antibiotic agents are in short supply and the need to conserve the remaining resources of effective antibiotics is of paramount importance. ${ }^{6} \mathrm{ABS}$ refers to coordinated interventions designed to improve and monitor the appropriate use of antibiotics by promoting the selection of the optimal antibiotic agent(s), dose, duration of therapy, and route of administration. The goals of ABS can be separated into: optimising therapy for individual patients, preventing overuse and misuse of antibiotics and minimising the development of resistance at patient and community health levels. The last six years have seen major shifts in policy towards combatting antibiotic resistance within the SA healthcare environment. This culminated in the National Department of Health's (NDoH) publication of SA's Antimicrobial Resistance National Strategy Framework 2014-24 in October 2014, at a Ministerial Antimicrobial Resistance (AMR) Summit. ${ }^{13}$ One of the strategic objectives of the framework 
was to 'promote appropriate use of antimicrobials in human and animal health' through ensuring access to safe, effective and affordable antimicrobials, institutionalising antimicrobial stewardship programmes across sectors, and addressing the use of antimicrobials in animal health and crop production. Parallel to this, and as part of the country's drive towards quality healthcare service delivery, specific $A B S$ and infection prevention control norms and standards were finalised and published for public comment in the Government Gazette, with a view of them becoming enforceable regulations. ${ }^{14} A$ need for $A B S$ implementation and monitoring in $S A$ was highlighted in a recent study where 47 private hospitals across $S A$ revealed that close to one in every 15 prescriptions contained inappropriately prescribed antibiotics and required intervention. ${ }^{15}$ In addition, the study highlighted the significant role of SA pharmacists in antibiotic stewardship, since their collective efforts had a substantial impact on reducing antibiotic consumption in their respective hospitals. This demonstrates the influence that pharmacist-led stewardship programmes can have in settings where infectious disease-trained specialists are limited, and when integrated as part of the multi-disciplinary team (MDT). ${ }^{15}$ Current research is being undertaken in public hospitals to assess the situation in this important sector.

\subsection{Roles and responsibilities of the antibiotic stewardship programme members in South Africa}

In the South African context, especially in the public sector, it is difficult to replicate resource-abundant stewardship models that are mostly driven by medical microbiology teams, infectious disease (ID) specialists and epidemiologists. ${ }^{16,17}$ Sustainable and effective antibiotic stewardship programmes (ASPs) largely depend on monitoring practices, teamwork in developing and implementing interventions, and organisational infrastructure. ${ }^{16}$ Consequently, it is critical in the SA context to utilise the existing resources of pharmacists, registered nurses (RNs) and other members of the healthcare team, who are well placed to coordinate anti-infective management and improve patient outcomes. ${ }^{18}$ For effective ASPs in SA, it is important to recognise the skills within Multidiscplinary team (MDT) that can support pharmacists to build effective ABS practices within the South African Antimicrobial Resistance National Strategy Framework ${ }^{13}$ and the associated Implementation Plan. ${ }^{14}$

For ASPs to be successful, each member of the healthcare team should wholeheartedly execute the requirements of their role and responsibilities, resulting in an impactful stewardship programme. Figure 1 displays the potential key roles of the various players in a stewardship programme.

\subsection{The role of the pharmacist in antibiotic stewardship}

Of the 13416 pharmacists registered in South Africa in 2015, around $37 \%$ are practicing in the public sector, a number which has steadily grown from around $16 \%$ since $2007 .{ }^{19}$ The average density of community pharmacies per head of population is around 0.61 per 10000 ; however, with the inclusion of primary care clinics this nearly reaches the WHO benchmark of one clinic per 10000 residents. ${ }^{20}$ Pharmacists can play a key role in the implementation of ASPs and although, to date, in SA this has not been a coordinated effort, recent evidence has shown success in the private sector. ${ }^{15}$

The distribution of healthcare human resources remains inequitable across the private (well-resourced) and public sectors

\begin{tabular}{l}
\multicolumn{1}{c}{ Microbiology } \\
- Preliminary microbiology \\
results and antibiotic \\
adjustment \\
- Final culture report and \\
antibiotic adjustment \\
- Identification of \\
antimicrobial resistance \\
- Advisory role on ASP \\
progress
\end{tabular}

\footnotetext{
Infection Control

- Triage and appropriate isolation

- Final culture report and antibiotic adjustment

- Identification of antimicrobial resistance

- Patient education

- Outpatient management

- Hand hygiene

- Isolation precautions
}

Nursing
- Triage and appropriate
isolation
- Accurate allergy history
- Early and appropriate cultures
- Timely antibiotic
administration
- Medication reconciliation
- Monitor and report on
progress
- Antibiotic dosing and
de-escalation
- Adverse events
- Monitor for changes in patient
condition
- IV to PO antibiotic switch
- Patient education
- Hand hygiene
Infection control precautions
- Case Management
- Monitor and report on
- IV to PO antibiotic switch
- Length of stay
Outpatient management

Pharmacy
- Accurate allergy history
- Medication reconciliation
- Preliminary microbiology
results and antibiotic
adjustment
- Antibiotic dosing and
de-escalation
- Adverse events
- Final culture report and
antibiotic adjustment
- IV to PO antibiotic switch
- Appropriate duration
- Ensuring medications are
available timeously for
administration
- Patient education
- Hand hygiene
- Infection control
precautions
- Pharmacokinetic and
dynamics adjustments

Figure 1. The attributes of function within current antimicrobial stewardship models ${ }^{18}$ 
(poorly resourced) although several initiatives introduced by the government have been implemented to draw healthcare professionals into the public sector. ${ }^{21}$

Further to this, formal training for $\mathrm{ABS}$ has not been defined, nor mandated in $S A$, and currently the role the pharmacist can play in the $A B S$ team would depend on the level of training that he/ she has received. As custodians of medicine, pharmacists are well placed to lead and drive the antibiotic stewardship initiative, not only through audit and data collection, but also through relationship-building with prescribers to influence crucial prescribing decisions. The scope of a stewardship pharmacist, across the various sectors, includes $22-24$ :

- Driving and coordinating the hospital ASP

- Antibiotic prescription review

- Drug expertise

- Evaluation of the appropriateness of prescribed antibiotics for given indications

- Dose optimisation

- Duration optimisation

- Monitoring, interpretation and follow-up of microbiology results

- Development, education and maintenance of clinical guidelines

- Measurement and tracking of stewardship-initiated interventions

- Measurement and tracking of compliance to institutionspecific guidelines

- Education and training of other healthcare providers

- Co-ordination of research on antibiotic stewardship

\subsection{Antibiotic preservation strategies}

Studies have shown that, rather than a single strategy in isolation, a combination of strategies described in this section, should be implemented, to have a sustainable impact on reducing the inappropriate use of antibiotics in hospital settings. ${ }^{25}$

\subsubsection{Formulary restrictions}

The formulary was one of the earliest methods used to directly influence antibiotic utilisation, and contain costs when several therapeutic agents from the same class became available. 26,27 Selecting antibiotics to include in a formulary requires the consideration of multiple factors, including: knowledge of local resistance patterns, avoiding agents with a similar spectrum of activity with no added benefits, drug costs and toxicity, amongst others. Formulary restriction protects agents that are more prone to resistance. Nevertheless, this strategy is rather broad and has not been proven to prevent overuse of available broad-spectrum antibiotics. ${ }^{27}$ However, this has depended on the acceptance and enforcement of formularies and treatment guidance. 28,29 Consequently, we believe it is necessary to combine formularies with other strategies to optimise appropriate use of antibiotics in the future. This includes regular reviews of antibiotic use against current guidance and resistance patterns, as well as de-escalation strategies. Periodic reviews of the formulary are needed, depending on changes in resistance patterns, availability of newer agents, new knowledge of side-effects of the recommended antibiotics, and other significant factors. ${ }^{27}$

Within the SA public sector, the implementation of the Essential Drugs Lists (EDLs) and Standard Treatment Guidelines (STGs) aids in the protection of antibiotics; however, this currently does not apply in the private sector.

\subsubsection{Education and guidelines}

Education is the most fundamental strategy by which prescribers may be influenced to adopt and maintain proficient prescribing practices ${ }^{27,29,30}$ and education forms a very important component of any ASP. ${ }^{26}$ Education can range from conference presentations, drug utilisation reviews and feedback, hospital pharmacy committee newsletters, student and provider teaching sessions, as well as the writing and dissemination of peerreviewed clinical guidelines for certain infectious diseases. ${ }^{31}$ The implementation of guidelines has been shown to improve the probability of appropriate initial antibiotic therapy, use of narrower-spectrum antibiotic regimens, earlier intravenous (IV)-to-oral switch therapy, and shorter duration of therapies (DOTs) - without negatively impacting clinical outcomes. ${ }^{32}$ Of importance, however, is that guidelines need to be regularly reviewed and updated, and most importantly, combined with other educational efforts, as well as direct interventions and monitoring of compliance to enhance their implementation in an endeavour to improve future care. ${ }^{27,28,33}$

\subsubsection{Prior approval/authorisation of protected antibiotics}

Prior approval/authorisation of protected antibiotics is another strategy that can be used to curtail antibiotic use, and requires justification for approval from the pharmacy or clinical head of the department for the use of certain antibiotics prior to prescription. ${ }^{32}$ This strategy has been identified as the most difficult to adhere to by prescribers, even though it can be the most effective in improving antibiotic prescribing. ${ }^{26} \mathrm{Approval}$ can be obtained in many ways, including: telephonic authorisations, antibiotic order forms and direct interactions with prescribers. ${ }^{34}$ However, there can be concerns with formulary or antibiotic restrictions that need to be considered and addressed before implementation. These include: delays in initiating therapy, prescriber pushback, and unintended increases in the use of unrestricted antibiotics. ${ }^{35}$ To help preserve antibiotic sensitivity, prior authorisation of selected antibiotics combined with education of clinicians on criteria for use can ensure appropriate prescribing of antibiotics. ${ }^{25}$ For effective implementation of this strategy, support from the hospital administration, supply of the necessary resources with persistent efforts from committed and trained personnel, together with continuous communication between pharmacists and prescribers, will be needed. ${ }^{32}$

\subsubsection{De-escalation/streamlining}

The persistent use of broad-spectrum antibiotics contributes to the increase in antibiotic-resistant pathogens. De-escalation/ 
streamlining is a method used to provide narrow-spectrum, targeted therapy following culture sensitivity results. ${ }^{33}$ The duration of antibiotic therapy can be reduced by shortening the course or terminating antibiotics if there is no evidence of infection. This is one of the most difficult strategies to implement and requires advanced training on the part of the pharmacist. The pharmacist must be aware of the full clinical picture of the patient, e.g. infection markers, response to treatment and other diagnostic measures before recommending such an intervention. ${ }^{27,33}$

\subsubsection{Prospective audit and feedback}

Prospective audit and feedback (PAF) is an intervention that reviews antibiotic prescriptions and engages the provider after an antibiotic is prescribed. Improvements on antibiotic use, reduction in antibiotic resistance and decline in Clostridium difficile infections (CDI) have been seen in PAF interventions with no negative influence on patient outcomes. One study, conducted by a clinical pharmacist and infectious diseases (ID) physician, led to a $22 \%$ decline in the use of parenteral broadspectrum antibiotics. ${ }^{32}$ In the ICU, PAF interventions showed a decrease in meropenem resistance and decreased $C D I$, without affecting mortality. ${ }^{32}$ The success of PAF is determined by the infrastructure and resources in place at an institution, as well as relationships and communication methods between pharmacists and prescribers. This process can be laborious and often requires ward bed-to-bed prescription surveillance. However, many interventions relating to PAF can be implemented at the point of dispensing in the pharmacy during evaluation and interpretation of the prescription chart. PAF improves antibiotic use and should be a fundamental component of any stewardship programme. ${ }^{32}$

\subsubsection{Pharmacokinetic and pharmacodynamic dosage optimisation}

Pharmacology is based on two principles namely, pharmacokinetics (PK) and pharmacodynamics (PD). Conventional antibiotic dosing and administration should be optimised in accordance with PK/PD principles for clinical efficacy. Emerging data shows that PK/PD-based approaches to antibiotic prescribing should not only be used to improve clinical outcomes, but also to reduce AMR through favourable dosing as these principles ensure ideal dosing concentrations and the avoidance of ineffective sub-therapeutic antibiotic exposure. ${ }^{36}$

In SA, PK/PD monitoring and adjustments should be implemented by hospitals for aminoglycosides and vancomycin due to inter-individual variation regarding PK/PD parameters. Contributing factors include the patient's age, weight, gender, organ function and disease state. Sub-therapeutic antibiotic exposure is a contributing cause of mortality within the criticallyill patient population suffering from severe systemic infections, which further stresses the importance of dose-optimisation via PK/PD principles for antibiotics. ${ }^{32,37,38}$

\subsection{Behavioural change/modifications}

Irrational prescribing is a global problem and interventions based on behavioural science may reduce this. There are many factors which can influence prescribing, which include cultural beliefs, socioeconomic factors, patient pressure, desire for clinical autonomy as well as "prescribing etiquette"; where prescribers are hesitant to alter prescriptions written by their colleagues. ${ }^{10}$ Prior to designing and implementing interventions to alter prescribing practices, it is important to conduct research regarding the factors which will influence prescribing practices in the specific institution. This will assist in determining what barriers will need to be addressed in order to successfully implement a particular intervention. Research has shown that senior colleagues and opinion leaders have more of an influence on prescribing practices than local policies and guidelines. Therefore, when designing interventions to change prescribing practice, it is imperative to involve senior clinicians and have their endorsement for any policy or guideline prior to implementation. ${ }^{16}$

A study done by Burger et al in SA stated that the most important intervention to decrease antibiotic resistance rates, is education. ${ }^{39}$ Clinicians need to receive education on antibiotic prescribing guidelines and policies in their institution, as this will also lead to enhanced effectiveness of interventions. ${ }^{40}$ Meeker et al also found that socially motivated interventions, like accountable justification and peer comparison, significantly reduce irrational antibiotic prescribing. Such interventions resulted in a greater reduction of inappropriate prescribing, than education or observation alone..$^{40}$

\subsection{Antibiotic stewardship measures}

The following antibiotic stewardship measures (Table I) have been suggested by the South African Best Care Always (BCA $)^{41}$ campaign and have been adopted by antibiotic stewards around the country.

\subsubsection{Quantifying antibiotic stewardship outcomes}

Currently there is no consensus with regards to which metric best quantifies antibiotic usage in hospitals. Metrics which may be used include Defined Daily Dose (DDD), Days of Therapy (DOT) and Length of Therapy (LOT). ${ }^{42}$ In ambulatory care, DDD and defined daily doses per 1000 inhabitants per day (DIDs), (DDDs/1000 inhabitants/day) are typically used. In Point Prevalence Studies (PPS), antibiotic bed-days are also used.

\subsubsection{DDD - Defined Daily Dose}

The most commonly-used metric is DDD. DDDs are determined by the WHO (World Health Organisation) and represent the average daily dose of an antibiotic in an average patient. ${ }^{43}$ The DDD is calculated as the total number of grams of antibiotic agent used divided by the number of grams in an average daily dose. ${ }^{42}$ Consequently, DDDs can be used to measure antibiotic consumption and can serve as a measure to determine the impact of an ASP. Antibiotic usage in hospitals can be expressed as DDDs per 100 or 1000 patient-days. The major advantage of 
Table I. Antibiotic stewardship measures ${ }^{41}$

\begin{tabular}{|c|c|c|c|}
\hline Challenge & Measure & $\begin{array}{l}\text { Pharmacist's Level of } \\
\text { Training }\end{array}$ & Interpretation \\
\hline $\begin{array}{l}\text { Empiric therapy without } \\
\text { confirmation }\end{array}$ & $\begin{array}{l}\text { Antibiotics prescribed without } \\
\text { appropriate cultures }\end{array}$ & Pharmacist & $\begin{array}{l}\text { Diagnostic microbiological cultures need to be } \\
\text { taken before initiation of antibiotic therapy, to } \\
\text { perform accurate 'drug-bug matching' and ensure } \\
\text { the patient is on the most appropriate antibiotic for } \\
\text { the infecting organism }\end{array}$ \\
\hline $\begin{array}{l}\text { Inappropriate choice of } \\
\text { antibiotic agent }\end{array}$ & 'Drug-bug matching' & Pharmacist & $\begin{array}{l}\text { Ensuring that each patient is on the correct } \\
\text { antibiotic agent as per culture sensitivity results }\end{array}$ \\
\hline $\begin{array}{l}\text { Inappropriate } \\
\text { combinations }\end{array}$ & $\begin{array}{l}\text { Double Gram-negative cover, } \\
\text { double Gram-positive cover, } \\
\text { double antifungal cover, or } \\
\text { double anaerobic agents }\end{array}$ & Pharmacist & $\begin{array}{l}\text { Generally, more than one agent from each class } \\
\text { should not be used simultaneously (i.e. two } \\
\text { anti-Gram-positive agents, two antifungals; } \\
\text { some antibiotic agents have anaerobic cover } \\
\text { and the addition of metronidazole is considered } \\
\text { double anaerobic cover; exceptions to this rule } \\
\text { include double Gram-negative cover for certain } \\
\text { Gram-negative infections, e.g. CRE }\end{array}$ \\
\hline $\begin{array}{l}\text { Polypharmacy } \\
\text { pertaining to antibiotics }\end{array}$ & $\begin{array}{l}\text { The concurrent administration } \\
\text { of four or more antibiotics }\end{array}$ & Pharmacist & $\begin{array}{l}\text { The concurrent administration of }>4 \text { antibiotic } \\
\text { agents is rarely considered appropriate. Duplication } \\
\text { of spectrum of activity is a concern when a higher } \\
\text { number of antibiotics are used concurrently and } \\
\text { thus requires review. }\end{array}$ \\
\hline Failure to de-escalate & $\begin{array}{l}\text { Streamlining therapy from } \\
\text { a broad-spectrum agent to } \\
\text { a narrower-spectrum agent } \\
\text { following culture sensitivity } \\
\text { results }\end{array}$ & Advanced level training & $\begin{array}{l}\text { Continuation of broad-spectrum therapy following } \\
\text { evidence of sensitivity to a narrower spectrum agent } \\
\text { can contribute to an increased risk of antibiotic } \\
\text { resistance }\end{array}$ \\
\hline $\begin{array}{l}\text { Excessive duration of } \\
\text { treatment }\end{array}$ & 7-14 days & Pharmacist & $\begin{array}{l}\text { Only in rare circumstance will treatment be } \\
\text { necessary for }>7 \text { days, i.e. bone sepsis, endocarditis, } \\
\text { known infections with MRSA, C. difficile, etc. }\end{array}$ \\
\hline IV-to-oral switch (IVOST) & $\begin{array}{l}\text { Agents which are } 100 \% \\
\text { bioavailable can be switched } \\
\text { interchangeably } \\
\text { Criteria for switching: } \\
\text { Improvement in clinical status } \\
\text { of patient } \\
\text { Tolerate oral feeds } \\
\text { Improvement of sepsis markers } \\
\text { Oral formulation available }\end{array}$ & Pharmacist & $\begin{array}{l}\text { They include: quinolones, voriconazole, linezolid, } \\
\text { clindamycin, erythromycin, clarithromycin, } \\
\text { metronidazole, fluconazole }\end{array}$ \\
\hline Hang-time & $\begin{array}{l}\text { Hang-time refers to the } \\
\text { difference in time between } \\
\text { the writing of the antibiotic } \\
\text { prescription and the time it is } \\
\text { first administered }\end{array}$ & Pharmacist & $\begin{array}{l}\text { There is } 7.6 \% \text { mortality with every } 1 \text {-hour delay in } \\
\text { antibiotic administration for patients with sepsis and } \\
\text { septic shock.Thus, hang-time should ideally not be } \\
\text { longer than an hour in duration, for the first dose of } \\
\text { antibiotics }\end{array}$ \\
\hline
\end{tabular}

[CRE: Carbapenem-Resistant Enterobacteriaceae]

the DDD is the ability to compare standardised doses amongst different hospitals, as well as different ambulatory care settings within and across countries. The disadvantage of the DDD is that it does not account for alternative dosing regimens, such as differences in renal function, gender, age or regimens that have been optimised by PK or PD dosing, so this can result in overestimation or underestimation of drug consumption. ${ }^{42,44}$ Certain shortcomings have been documented with the DDD method, such as the fact that it does not apply to the paediatric population and the administered daily dose usually differs significantly from the WHO approved DDD because the minimum inhibitory concentration (MIC) for various organisms has increased over time due to the emergence of bacterial resistance. ${ }^{45}$ However, in 2016 the National Department of
Health $(\mathrm{NDoH})$ endorsed the use of DDD in the surveillance of antibiotic consumption, as part of the activities in an ASP outlined for public sector hospitals. The data in each province from all central, secondary level, and high-throughput district level hospitals must be presented as defined daily doses per hundred patient days (DDD/100 patient days).

\subsubsection{DOT - Days of Therapy}

An alternative measure of antibiotic consumption is DOT. DOTs are expressed as the administration of a single agent on a given day without taking into consideration the doses administered or the strength. For example, if a patient receives one antibiotic for three days, it equals three DOTs. Similarly, if a patient received three different antibiotics for three days, it 
would equal nine DOTs. Both DDDs and DOTs are useful for interfacility comparisons and facility-level monitoring of antibiotic consumption. ${ }^{42}$ Important advantages of DOTs are that they are unaffected by the dosage regimen the patient is receiving, can be used for both adult and paediatric populations, and can facilitate a comparison between different classes of antibiotics within the same institution. Conversely, a disadvantage is that DOT may not give an accurate representation of antibiotics that are administered multiple times on the same day. ${ }^{32,42,46}$

\subsubsection{LOT - Length of Therapy}

Length of Therapy (LOT) represents the number of days a patient receives antibiotic therapy. LOT does not take into consideration the number of antibiotics the patient received. For example, if a patient receives three different antibiotics for three days, it would equal three LOTs. When compared to DOT, LOT gives a more accurate estimation of the duration of antibiotic therapy. ${ }^{42}$ An important activity for a stewardship programme involves recommendations for LOT based on patient-specific factors. Developing written guidelines with specific suggestions for duration, including LOT recommendations as part of the preauthorisation or prospective audit and feedback processes, or specifying duration at the time of antibiotic ordering, are examples of suitable approaches that may be considered. . $^{32,46}$

\subsection{Monitoring antibiotic stewardship strategies}

\subsubsection{Local prevalent sensitivities and resistance patterns}

An important aspect of ASPs is to identify the current local pathogens that are prevalent in the institution during the formulation of policies. Simply following the national guidelines may not always be the best option, because not all hospitals will experience the same pathogen prevalence and resistance patterns. The pharmacist should play an active role in identifying which antibiotics are most frequently used, and sometimes overused, and can collaborate with microbiologists who can provide input on hospital-specific pathogens and resistance patterns. $^{42}$

\subsubsection{Antibiograms}

An antibiogram shows an overall profile of microorganism susceptibility to a specific antibiotic in the hospital. Institutional antibiograms will help to develop empiric antibiotic therapy guidelines, which become vital whenever there are delays in obtaining microbiology results. ${ }^{47}$ Stratified antibiograms include parameters, such as patient location, population and infection site, and can assist in optimising treatment recommendations and guidelines. For example, certain resistant organisms may be more prevalent in intensive care units (ICU) than in non-ICU settings. ${ }^{32}$

When constructing an antibiogram, it is important to control the source of the isolates. For example, isolates from outpatient samples should be excluded when constructing an antibiogram for inpatients, and vice versa. Microbiologists should always be consulted when trying to establish an antibiogram for a hospital, because strict criteria and rules need to be applied to ensure that the data reflected in the antibiogram is valid.

The Infectious Diseases Society of America (IDSA) supports the use of stratified antibiograms over non-stratified antibiograms, to assist in the development of relevant guidelines. Whilst evidence that stratified antibiograms promotes improved empiric antibiotic therapy is limited, stratification can reveal important susceptibility differences. ${ }^{32}$

\subsubsection{Infection control}

The transmission of highly-resistant bacteria from one patient to the next, or from patients to healthcare workers and vice versa, amplifies the problem of resistance. Figure 2 depicts the strategic objectives, pertaining to infection prevention and control, per the SA AMR framework strategy..$^{13}$

\section{Strategic objective 3: Enhance infection prevention and control}

\section{Sub-objective 3.1: Prevent new}

\section{infections}

Prevention of infection through wide-reaching vaccination programmes and improvements in water and sanitation are also key prevention strategies to reduce AMR. Optimising vaccine uptake to prevent infection, and therefore the need for antibiotics, is an important tool in preventing resistance. This will be implemented though primary prevention and promotion activities within the public District Health System and private health systems.
Sub-objective 3.2: Prevent and control the spread of resistant microorganisms Infection Prevention and Control (IPC) in the healthcare facility is a core component of overall quality improvement, which if strengthened holistically, will directly and indirectly address the current problem of AMR. Controlling the spread of resistant microbes to patients and the workforce involves rapid identification and isolation of patients with resistant organisms, access to personal protective equipment, and most importantly, improvement in hand hygiene. The multi-modal WHO intervention 'Five Moments for Hand Hygiene' will be adopted in all South African healthcare facilities as the gold standard for hand hygiene and the implementation of sustained campaigns.

A key enabler to effective infection control includes sufficient, suitably qualified and competent IPC practitioners with defined core skills and responsibilities for supporting the implementation of control measures in the facility.

Similarly, although more difficult to implement, interventions focused on community awareness of basic infection prevention and hand hygiene measures may provide long-term benefits and will require a national awareness campaign and reinforcement through the direct community engagement of Community Healthcare Workers.

Figure 2. The strategic objectives, pertaining to infection prevention and control, per the SA AMR framework strategy ${ }^{13}$ 
They key to minimising such transmissions, is careful attention to infection control practices. Key elements of such practices, per the WHO, include ${ }^{48}$ :

- Appropriate barrier precautions for high risk patients (hand washing, wearing gloves and gowns)

- Appropriate isolation procedures depending on patient risk

- Adequate sterilisation and disinfection of supplies and equipment

- Use of aseptic techniques for medical and nursing procedures

- Training of healthcare personnel in appropriate sterile techniques and infection control procedures

- Maintaining appropriate disinfection and sanitary control of the hospital environment, including the air

- Provision of sufficient basins and hand/paper towels to regularly wash hands between patient contacts

- Recognition, investigation and management of outbreaks or clusters of infection

- Surveillance of hospital-acquired infections (HAI) and AMR

- Guideline development together with the standardisation of effective prevention practices and the implementation thereof (i.e. standard operation procedures)

- Assessment with feedback regarding IPC.

Pharmacist should adhere to theappropriate infection prevention and control protocols of the hospital whilst conducting bedside surveillance for $A B S$ in the wards. This should include being 'Bare Below the Elbows' and adhering to hand hygiene practices between patients and whenever applicable.

\section{Conclusion}

AMR has become a very real and concerning threat to modernday healthcare practice. The effective management thereof requires a decisive and multifaceted approach, which involves several members of the multidisciplinary healthcare team. The pharmacist is ideally positioned, and needs to play a central role within this setting, to facilitate and coordinate the implementation and monitoring of antimicrobial stewardship programmes (ASPs). Effective ASPs are required to preserve antibiotics via measures such as formulary restrictions, education and guidelines, pre-authorisations, de-escalation procedures, audits, and dose optimisation strategies. The successful implementation of such programmes will be greatly dependent upon the effective quantification and monitoring of stewardship outcomes.

\section{References}

1. De Luca $M$, Donà $D$, Montagnani $C$, et al. Antibiotic prescriptions and prophylaxis in Italian children. Is it time to change? 2016. Data from the ARPEC Project. De Socio GV, ed. PLoS ONE , 11(5):e0154662. DOI: 10.1371/journal. pone. 0154662.

2. World Health Organization (WHO) 2014. Antimicrobial resistance: global report on surveillance. Available online: http://www.who.int/drugresistance/ documents/surveillancereport/en/ Accessed 28 May 2017.

3. Hwang TJ, Powers JH, Carpenter D, Kesselheim AS. 2015. Accelerating innovation in rapid diagnostics and targeted antibacterials. Nature Biotechnology, 33, pp. 589-590.
4. Laxminarayan R, Matsoso $P$, Pant $S$, et al. 2016. Access to effective antimicrobials: a worldwide challenge. Lancet, 387(10014), pp. 168 - 75.

5. Van Boeckel TP, Gandra S, Ashok A, et al. 2014. Global antibiotic consumption 2000 to 2010: an analysis of national pharmaceutical sales data. The Lancet Infectious Diseases, 14(8), pp. 742-50.

6. O'Neill J. 2016. Tackling drug-resistant infections globally: Final report and recommendations. The Review on Antimicrobial Resistance. Available online: https://amr-review.org/sites/default/files/160518_Final\%20paper_ with\%20cover.pdf Accessed 29 May 2017.

7. Hoffman SJ, Outterson K. 2015. Introduction: What will it take to address the global threat of antibiotic resistance? The Journal of Law, Medicine \& Ethics, 43(3), pp, 6-11.

8. Barlam TF, Gupta K. Antibiotic resistance spreads internationally across borders. The Journal of Law, Medicine \& Ethics. 2015;43 Suppl 3:12-6.

9. Nguyen KV, Thi Do NT, Chandna A, et al. 2013. Antibiotic use and resistance in emerging economies: a situation analysis for Viet Nam. BMC public health, 13(1158). Available online at: http://download.springer.com/static/pdf/452/ art\%253A10.1186\%252F1471-2458-13-1158.pdf?originUrl=http\%3A\%2F\%2Fb mcpublichealth.biomedcentral.com\%2Farticle\%2F10.1186\%2F1471-2458-131158\&token $2=\exp =1496039066$ acl $=\% 2 F$ static\% $2 F p d f \% 2 F 452 \% 2 F$ art $\% 25253 \mathrm{~A}$ 10.1186\%25252F1471-2458-13-1158.pdf* $\sim \mathrm{hmac}=\mathrm{f} 4 \mathrm{fab} 541 \mathrm{~d} 5 \mathrm{fb} 1 \mathrm{cebc} 42 \mathrm{abc6ae}$ cc415533d48028008b1c791513b37d4dc499f83 Accessed 29 May 2017.

10. Rezal RSMd, Hassali MA, Alrasheedy AA, et al. Godman B. 2015. Physicians' knowledge, perceptions and behaviour towards antibiotic prescribing: a systematic review of the literature. Expert Review of Anti-Infective Therapy, 13(5), pp. 665-80.

11. Mohamoud SA, Yesuf TA, Sisay EA. 2016. Utilization assessment of surgical antibiotic prophylaxis at Ayder Referral Hospital, Northern Ethiopia. Journal of Applied Pharmacy, 8(220) DOI: 10.4172/1920-4159.1000220.

12. Infectious Diseases Society of America (IDSA). 2011. Combating antimicrobial resistance: Policy recommendations to save lives. Clinical Infectious Diseases, 52(S5), pp, S397-S428.

13. National Department of Health. Antimicrobial resistance: National strategy framework 2014-2024. Available online at: https://www.health-e.org.za/ wp-content/uploads/2015/09/Antimicrobial-Resistance-National-StrategyFramework-2014-2024.pdf Accessed 29 Jan 2017.

14. National Department of Health, South Africa. Implementation plan for antimicrobial resistance national strategy framework 2014-2019. Available online at: http://www.health.gov.za/index.php/antimicrobial-resistance Accessed 20 July 2016.

15. Brink A, Messina A, Feldman C, et al. 2016. Antimicrobial stewardship across 47 South African hospitals: an implementation study. The Lancet Infectious Diseases, 16(9), pp. 1017-1025.

16. Charani E, Edwards R, Sevdalis N, et al. 2011. Behaviour change strategies to influence antimicrobial prescribing in acute care: A systematic review. Clinical Infectious Diseases, 53(7), pp. 651-662. DOl: 10.1093/cid/cit212.

17. Olans RN, Olans RD, DeMaria A. 2016. The critical role of the staff nurse in antimicrobial stewardship - unrecognized, but already there. Clinical Infectious Diseases, 62(1), pp. 84-89. DOI: 10.1093/cid/civ697.

18. Schellack N, Pretorius R, Messina AP. 2016. 'Esprit de corps': Towards collaborative integration of pharmacists and nurses into antimicrobial stewardship programmes in South Africa. South African Medical Journal, 106(10), pp. 973-974. DOI: 10.7196/SAMJ.2016.v106i10.11468.

19. Gray A, Riddin J, Jugathpal J. 2016. Health care and pharmacy practice in South Africa. The Canadian Journal of Hospital Pharmacy, 69(1), pp. 36-41.

20. Ward K, Sanders D, Leng H, Pollock AM. 2014. Assessing equity in the geographical distribution of community pharmacies in South Africa in preparation for a national health insurance scheme. Bulletin of the World Health Organization, 92(7), pp. 482-489. doi:10.2471/BLT.13.130005.

21. Van Rensburg HC. 2014. South Africa's protracted struggle for equal distribution and equitable access - still not there. Human Resources for Health, 12(26). DOI:10.1186/1478-4491-12-26.

22. Broom A, Plage S, Broom J, et al. 2016. A qualitative study of hospital pharmacists and antibiotic governance: Negotiating interprofessional responsibilities, expertise and resource constraints. BMC Health Services Research. DOI:10.1186/s12913-016-1290-0.

23. Van der Horst D. 2016. Antimicrobial stewardship: What if I don't work in the hospital? Michigan Pharmacists. Available online at: http://www. 
michiganpharmacists.org/Portals/0/Derek\%20VanderHorst\%20Powerpoint. pdf?ver=2016-09-26-112831-417 Accessed 29 May 2017.

24. Erickson AK. 2016. Hospital pharmacists are essential to antimicrobial stewardship. Pharmacy Today: Health Systems Edition. Pp. 6-7.

25. Reed EE, Stevenson KB, West JE, et al. 2013. Impact of formulary restriction with prior authorization by an antimicrobial stewardship program. Virulance, 4(2), pp. 158-162.

26. Fishman N. 2006. Antimicrobial stewardship. American Journal of Infection Control, 34(5), pp. S55-S63.

27. Paskovaty A, Pflomm J, Myke N, Seo S. 2005. A multidisciplinary approach to antimicrobial stewardship: evolution into the 21 st century. International Journal of Antimicrobial Agents, 25(1), pp. 1-10.

28. Bjorkhem-Bergman L, Andersen-Karlsson E, Laing R, et al. 2013. Interface management of pharmacotherapy. Joint hospital and primary care drug recommendations. European Journal of Clinical Pharmacology, 69(1), pp. 73-78.

29. Gustafsson LL, Wettermark B, Godman B, et al. 2011. The 'wise list' - a comprehensive concept to select, communicate and achieve adherence to recommendations of essential drugs in ambulatory care in Stockholm. Basic \& Clinical Pharmacology \& Toxicology, 108(4), pp. 224-233.

30. Wettermark B, Godman B, Jacobsson B, Haaijer-Ruskamp FM. 2009. Soft regulations in pharmaceutical policy making: an overview of current approaches and their consequences. Applied Health Economics and Health Policy, 7(3), pp. 137-147.

31. Suleman F, Meyer H. 2012. Antibiotic resistance in South Africa; your country needs you. South African Pharmacy Journal, 79(5).

32. Barlam TF, Cosgrove SE, Abbo LM, et al. 2016. Implementing an Antibiotic Stewardship Program: Guidelines by the Infectious Diseases Society of America and the Society for Healthcare Epidemiology of America. Clinical Infectious Diseases, 62(10), pp, e51-e77.

33. Dellit T. 2007. Summary of the Infectious Diseases Society of America and the Society for Healthcare Epidemiology of America Guidelines for Developing an Institutional Program to Enhance Antimicrobial Stewardship. Infectious Diseases in Clinical Practice, 15(4), pp.263-264.

34. Owens RC (Jr). 2008. Antimicrobial stewardship: concepts and strategies in the 21st century. Diagnostic Microbiology and Infectious Disease, 61, pp. 110-128.

35. Furst J, Cizman M, Mrak J, et al. 2015. The influence of a sustained multifaceted approach to improve antibiotic prescribing in Slovenia during the past decade: findings and implications. Expert Review of Anti-Infective Therapy, 13(2), pp. 279-289.

36. Abdul-Aziz M, Lipman J, Mouton JW, et al. 2015. Applying pharmacokinetic/ pharmacodynamic principles in critically ill patients: optimizing efficacy and reducing resistance development. Seminars in Respiratory and Critical Care Medicine, 36(1), pp. 136-153.

37. Blot SI, Pea F, Lipman J. 2014. The effect of pathophysiology on pharmacokinetics in the critically ill patient - Concepts appraised by the example of antimicrobial agents. Advanced Drug Delivery Reviews, 77, pp. 3-11.

38. Wong G, Sime FB, Lipman J, Roberts JA. 2014. How do we use therapeutic drug monitoring to improve outcomes from severe infections in critically ill patients? BMC Infectious Diseases, 14:288. DOI:10.1186/1471-2334-14-288.

39. Burger M, Fourie J, Loots D, et al. 2016. Knowledge and perceptions of antimicrobial stewardship concepts among final year pharmacy students in pharmacy schools across South Africa. Southern African Journal of Infectious Diseases, 31(3), pp. 84-90.

40. Meeker D, Linder JA, Fox CR, et al. 2016. Effect of behavioral interventions on inappropriate antibiotic prescribing among primary care practices: $\mathrm{A}$ randomized clinical trial. Journal of American Medical Association, 315(6), pp. 562-570.

41. Bestcare.org.za. 2016. Best Care... Always! - home. [online] Available online at: http://www.bestcare.org.za Accessed 5 Dec. 2016.

42. Doron S, Davidson LE. 2011. Antimicrobial stewardship. Mayo Clinic Proceedings. 86(11) pp. 1113-1123.

43. World Health Organization (WHO). WHO Collaborating Centre for Drug Statistics Methodology. ATC/ DDD Index. Available online at: https://www.whocc.no Accessed 29 May 2017.

44. Polk RE, Fox C, Mahoney A, et al. 2007. Measurement of adult antibacterial drugs use in 40 US hospitals: Comparison of Define Daily Dose and Days of Therapy. Clinical Infectious Diseases, 44(5), pp. 664-670. DOI: https://doi. org/10.1086/511640.

45. Bansal D, Mangla S, Undela K, et al. 2016. Measurement of adult antimicrobial drug use in tertiary care hospital using Defined Daily Dose and Days of Therapy. Indian Journal Of Pharmaceutical Sciences, 76(3), pp. 211-217.

46. Schechner V, Temkin E, Harbarth S, et al. 2013. Epidemiological interpretation of studies examining the effect of antibiotic usage on resistance. Clinical Microbiology Reviews, 26 (2), pp. 289-307.

47. Massele A, Tiroyakgosi C, Matome M, et al. 2017. Research activities to improve the utilization of antibiotics in Africa. Expert Review Of Pharmacoeconomics \& Outcomes Research, 17(1), pp. 1-4.

48. Guidelines on core components of infection prevention and control programmes at national and acute healthcare facility level. 2016. World Health Organization (WHO). Available online at: http://passthrough fw-notify.net/download/376002/http://apps.who.int/iris/bitstr eam/10665/251730/1/9789241549929-eng.pdf?ua=1 Accessed 29 May 2017. 